\title{
Multi-Party Energy Management for Clusters of Roof Leased PV Prosumers: A Game Theoretical Approach
}

\author{
Nian Liu ${ }^{1, *}$, Cheng Wang ${ }^{1}$, Xinhao Lin ${ }^{1}$ and Jinyong Lei ${ }^{2}$ \\ 1 State Key Laboratory of Alternate Electrical Power System with Renewable Energy Sources, \\ North China Electric Power University, Changping District, Beijing 102206, China; \\ wangcheng@ncepu.edu.cn (C.W.); xinhao@ncepu.edu.cn (X.L.) \\ 2 Electrical Power Research Institute, China Southern Power Grid (CSG), Guangzhou 510080, China; \\ leijy@csg.cn \\ * Correspondence: nianliu@ncepu.edu.cn; Tel.: +86-10-8079-0940
}

Academic Editor: George Tsatsaronis

Received: 12 April 2016; Accepted: 6 July 2016; Published: 13 July 2016

\begin{abstract}
The roof-leased business mode is an important development method for the distributed photovoltaic (PV) systems. In this paper, the benefits of the PV energy are considered in a PV cluster (PVC) consisting of a certain number of prosumers and a PVC operator (PVCO). In order to distribute the benefits, a multi-party energy management method for the PVC is proposed, including an internal pricing model and a demand response (DR) model. First, the dynamic internal pricing model for the trading between PVCO and prosumers is formulated according to the economic principle of demand and supply relation. Moreover, in order to improve the local consumption of PV energy, the DR model is formulated as a non-cooperative game among the prosumers. Meanwhile, the existence and uniqueness of the Nash Equilibrium (NE) are proved, and a distributed solving algorithm is introduced to approach the NE solution. Finally, the PVC including four prosumers is selected as the study object, the results have shown that the internal pricing model and DR model can improve the benefit of both prosumers and PVCO, as well as the local consumption of PV energy.
\end{abstract}

Keywords: photovoltaic cluster; dynamic price; demand response; non-cooperative game; Nash Equilibrium

\section{Introduction}

With the development of distributed photovoltaic (PV) systems, PV prosumers (i.e., commercial buildings, residential houses, factories, etc.) have developed widely. Gradually, the concept of the PV cluster (PVC) has also emerged, which consists of a number of neighboring prosumers that are connected to the same microgrid or distribution grid [1,2]. In the PVC, the prosumers with different load profiles can share the produced PV energy and reduce the power fed to the utility grid [3]. In order to facilitate the energy sharing, an internal price is required for billing between the PVCO and prosumers [4,5]. Moreover, if the prosumers have flexible load resources, the dynamic internal pricing can be utilized as a demand response (DR) tool that regulates their power consumption behaviors to enhance the local consumption of PV energy [6,7]. As each prosumer is an independent and autonomous entity, the multi-party energy management of the PVC is an important component for realizing the optimal operation.

Until recently, there have been numerous studies focused on the related problems. Generally, these studies can be divided into two categories. The first category of methods are those that set the internal pricing based on the marginal cost of the distributed generator. In order to optimally manage the microgrid resources on both the generation and demand side, [7] presents a stochastic resource planning strategy to improve the operation efficiency based on an internal pricing strategy. Considering 
the uncertainty of marginal revenue and marginal cost in the inner power market of the microgrid, [8] proposes a price setting strategy that can deal with the power fluctuations caused by the wind and solar energy. Based on the marginal cost of power generation, optimal dispatching for interconnected microgrids is proposed in [9] by setting the dynamic selling price of electricity. Considering the relationship between the cost of distributed generators and feed-in tariff, [10] formulates an optimal scheduling model for the industrial enterprise microgrid. For the aforementioned studies, although the marginal cost of each power generation unit could be helpful to set a fair and reasonable internal price, it could not be used for setting the electricity price of PV energy, due to the uncertainty power output and zero fuel consumption.

The second category of methods mainly focuses on the energy management with renewable energy sources by using dynamic pricing mechanisms. For instance, [11] uses a real-time dynamic pricing strategy to set the internal buying and selling price of electricity, and thus, the profit of service providers can be maximized on the basis of reasonable revenue of end users. Moreover, [12] proposes an efficient and competing mechanism to solve the demand and supply problem in a microgrid with many consumers and suppliers, which is modeled as a Potluck Problem. Internal pricing is also used in [13] to determine the energy management scheme for a smart community combined with distributed energy resources. A non-cooperative Stackelberg Game is proposed to achieve optimal solutions for both entities. Furthermore, as also proposed for a smart community, [14] assumes that each residential unit has an energy storage system and they can share them with the coordinator to improve the benefits of both sides, and an auction-based mechanism is designed to determine the internal prices of renewable energy sources.

In this paper, we focus on the multi-party energy management problem of roof-leased PVCs. Generally, the roof-leased mode is a new emerging business mode for PV development, which means that the PVCO has invested on the PV systems and installed them on the roofs leased by the prosumers. By using this business mode, the PVCO can get profit from the produced electricity while the prosumers can save the power consumption cost with a lower electricity price. However, the supply and demand in the PVC cannot be balanced in most of the time. That is, the PVC should also interact with the utility grid to export the surplus PV energy or import the insufficient energy for load demand. Therefore, there are three types of entities involved in this energy management problem, including the PV prosumers, the PVCO, and the utility grid. According to the feed-in-tariff and electricity prices of many countries, the export and import prices of PV energy are constant for a certain time period. In this regard, the PVCO acts as a coordinator to optimize the operation of PVC by setting the internal prices, which under the constraints of feed-in-tariff and electricity price of utility grid. Moreover, prosumers can be regarded as an autonomous entity with a flexible load to be scheduled with the variation of internal prices. The utility grid is only regarded as the balancer in the problem. To the best of our knowledge, the multi-part energy management of the PVC is a new problem with the development of the roof-leased mode of PV prosumers, which is different from the aforementioned studies.

To this end, this paper proposes a multi-party energy management method for clusters of roof-leased PV prosumers. The main contributions are as follows: (1) according to the feed-in tariff policy of distributed PV in China, a dynamic internal pricing model for PVC under the roof-leased mode is proposed; (2) after analyzing the DR behavior of prosumers, a non-cooperative game among prosumers is formulated, and the existence and uniqueness of the Nash Equilibrium (NE) are also proved; (3) an iterative algorithm is proposed to approach the NE, which can be adopted by the prosumers in a distributed and autonomous manner.

The remainder of the paper is organized as follows: Section 2 introduces the structure and function of PVC. Sections 3 and 4 propose the economic model and DR model of PVC, respectively. Section 5 provides the case study and the conclusion is given in Section 6. 


\section{Structure and Function of the PVC}

According to the feed-in-tariff scenario, all the PV output power is encouraged to be consumed locally inside the PVC. If there is a PV power surplus, the excess PV energy is sold to the utility grid by the PVCO. Otherwise, if the PV power is insufficient, electricity is purchased from the utility grid by PVCO to meet the demand of prosumers.

As shown in Figure 1, the PVC contains a certain number of prosumers, who have leased roofs to the PVCO for the installation of PV panels. The PV power generated is assumed to be owned by the PVCO and the PVCO is assumed to supply cheaper energy to the prosumers. The prosumers are connected in the same microgrid or distribution grid. Meanwhile, every prosumer has a user energy management system (UEMS) for collecting local information, and participates in DR to promote his utility by scheduling of power consumption, according to the electricity price $[15,16]$.

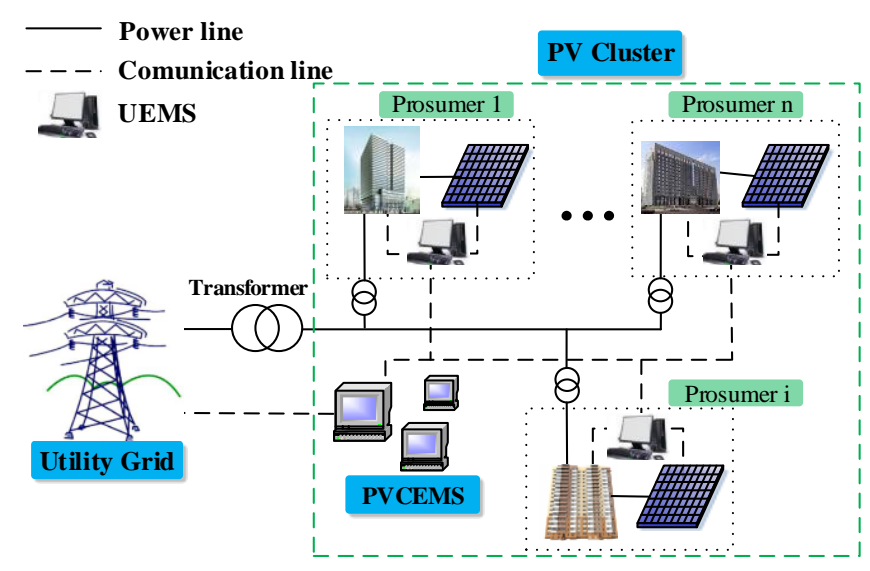

Figure 1. Structure of a PVC.

As a coordinator, the PVCO is obligated to decide the real-time internal prices based on the supply and demand conditions of the PVC. In order to facilitate the management, the energy management system (EMS) of PVC has two tasks: (1) it forecasts the PV energy of PVC, and distributes the forecasted value to all the prosumers; (2) collects the scheduled energy consumption of prosumers, and distributes other prosumers' total energy consumption to each prosumer.

\section{Economic Model of the PVC}

\subsection{Dynamic Internal Pricing Model}

According to the feed-in-tariff energy policy used in many countries, the feed-in price of PV energy is much lower than the electricity price of the utility grid $[17,18]$. Therefore, an internal pricing mechanism needs to be developed to support the inner electricity trading of the PVC, and be beneficial for both PVCO and prosumers. Hence, the internal selling price should be higher than the feed-in price of PV energy, and internal buying price of prosumers should be lower than the utility electricity price.

Trading prices and relationships of PVCO-utility grid and PVCO-prosumers are shown in Figure 2. PVC needs to be balanced by the utility grid with buying price $\lambda^{b u y}$ and selling price $\lambda^{\text {sell }}$. Meanwhile, all the prosumers would agree to pay the consumed electricity with the internal buying price $p r$.

Power consumptions of $N$ prosumers can be expressed as:

$$
x \triangleq\left\{x_{1}, x_{2}, \ldots, x_{N}\right\}
$$


where $x_{n}$ is the power consumption of prosumer $n$. We denote the predicted PV energy of the PVC is $P_{P V}$, the net energy charge (NEC) [10] between PVC and utility grid can be expressed as:

$$
N E C=\left\{\begin{array}{l}
\lambda^{\text {buy }}\left(\sum_{n=1}^{N} x_{n}-P_{P V}\right), \mid P_{P V}<\sum_{n=1}^{N} x_{n} \\
\lambda^{\text {sell }}\left(\sum_{n=1}^{N} x_{n}-P_{P V}\right), \mid P_{P V} \geqslant \sum_{n=1}^{N} x_{n}
\end{array}\right.
$$

where positive NEC means the energy buying cost that PVC pays to the utility grid, and negative NEC means the energy selling profit that PVC received from the utility grid.

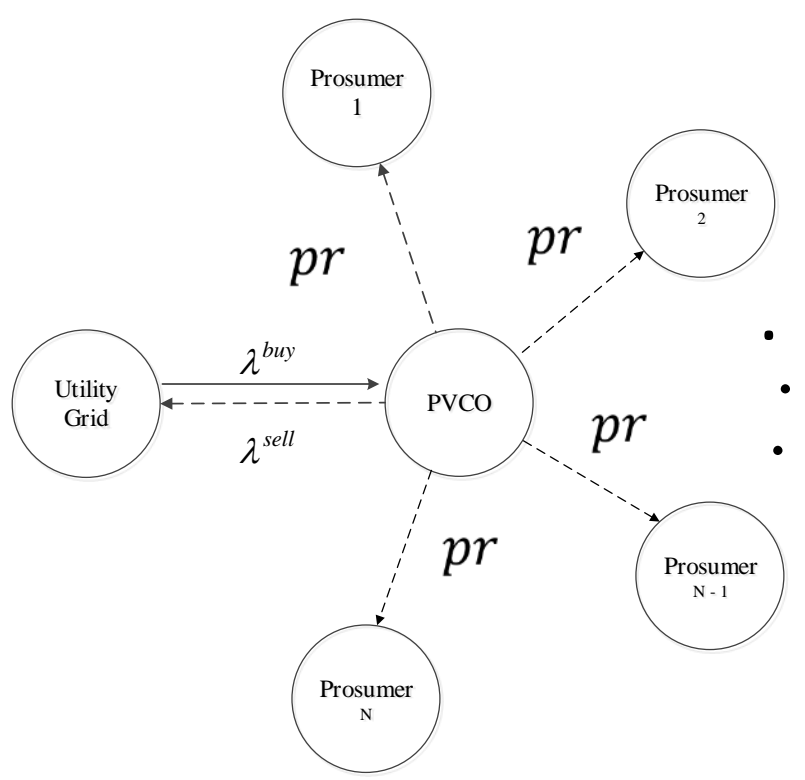

Figure 2. Relationship and prices of energy exchanges among prosumers, PVCO and the utility grid.

According to the economic principle, the price of the goods is determined by the supply and demand [19]. For the PVC, the power from the utility grid can be seen as infinite, but the limited PV power sold by PVCO can be seen as a scarce commodity with a lower price. In this regard, demand and supply ratio (DSR) is defined as:

$$
D S R=\frac{\sum_{n=1}^{N} x_{n}}{P_{P V}}
$$

To encourage the prosumers to utilize the PV energy, a pricing model is needed. Before designing the dynamic pricing model, we must consider three basic principles: (i) the internal prices should be bounded between the feed-in tariff and the electricity price of the utility grid; (ii) PV power should be utilized in the PVC as much as possible, and the dynamic pricing model can be used to encourage the consumption when the PV power is at a surplus; (iii) economic balance should always be guaranteed by the PVCO. Following these principles, we have added the detailed modeling of internal prices given in Appendix. Thus, the internal buying price of prosumers can be formulated as:

$$
p r=f(D S R)=\left(\lambda^{b u y}-\lambda^{\text {sell }}\right) e^{\frac{-\alpha}{D S R}}+\lambda^{\text {sell }}
$$

where $\alpha$ is the adjustment parameter of the price level, $0<\alpha \leqslant 1$.

According to the internal buying price, DSR at any time period can be obtained by Equation (3). If the PV power is very insufficient or even close to zero, the internal price $p r=\lambda^{b u y}$, as the $D S R \rightarrow+\infty$. If the load of prosumers is very small and PV power is at a surplus, internal price 
$p r=\lambda^{\text {sell }}$, as the $D S R \rightarrow 0$. As a result, the internal buying price could ensure prosumers get power supply at a price lower than $\lambda^{\text {buy }}$, which accordingly saves them money.

As the PV energy could be sold to prosumers by PVCO, in combination of NEC and internal buying price $p r$, the internal selling price of PV energy $p r_{P V}$ can be obtained as:

$$
\operatorname{pr}_{P V}=g(D S R)=\left\{\begin{array}{c}
p r \cdot D S R+\lambda^{\text {sell }}(1-D S R), \mid 0 \leqslant D S R \leqslant 1 \\
p r \cdot D S R-\lambda^{\text {buy }}(D S R-1), \mid D S R>1
\end{array}\right.
$$

According to Equations (4) and (5), relations between internal prices and DSR are shown in Figure 3 . When $0 \leqslant D S R \leqslant 1$, it means that the PV energy is more than the load demand. If we assume that the PV energy is constant, the DSR will increase with the load demand of prosumers. That is, the surplus PV energy fed to the utility grid reduces with the increasing DSR. Therefore, the internal buying price and internal selling price are both increasing with the DSR. When DSR $>1$, it means that the PV energy is less than the load consumption. The shortage of PV energy increases with the increased DSR and it needs to be bought from the utility grid at price $\lambda^{\text {buy }}$. According to the price design principles, even if the internal buying price increases with the DSR, it shouldn't be higher than the electricity price of the utility grid. Thus, in order to ensure the economic balance of PVC, the internal selling price $p r_{P V}$ has to be decreased with the DSR.

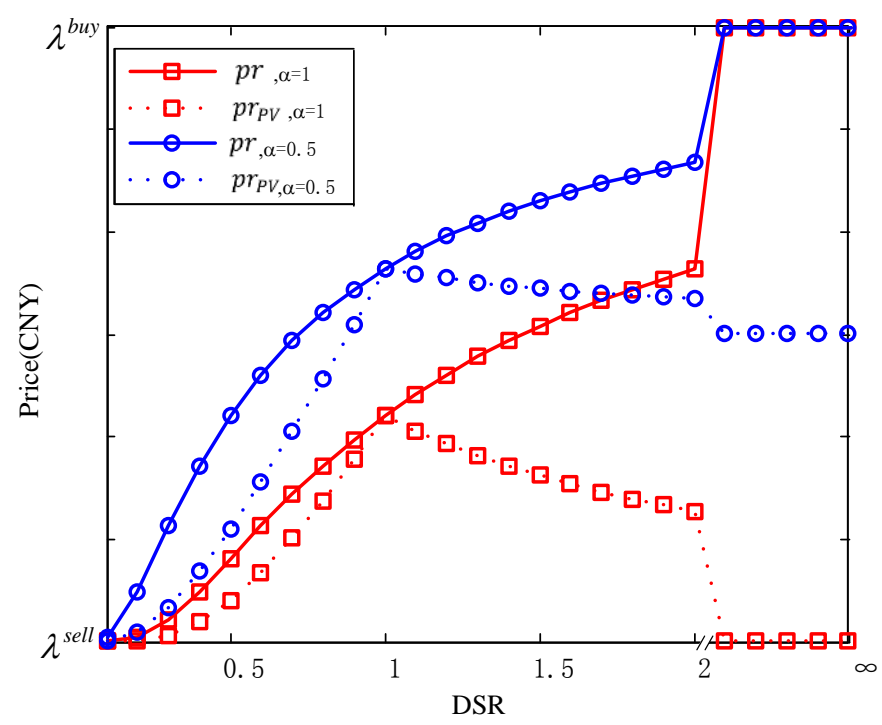

Figure 3. The relationship between internal buying price and PV selling price with DSR.

Meanwhile, internal prices show different characteristics with the increase of $\alpha$. In Figure 3, the internal prices are lower with a bigger value of $\alpha$, which is more beneficial to prosumers, whereas the internal prices are higher with a smaller value of $\alpha$, which is more beneficial to the PVCO. In the practical applications, PVCO can consider the cost of investment and negotiate with prosumers to set a value for $\alpha$, which should be acceptable to both sides.

\subsection{Prosumer's Utility Model}

It is proposed in [13] that the integral utility achieved by each prosumer consists of the utility and cost of the power consumption. Generally, the PVCO should be the operator that buys energy from and sells energy to the prosumers. In this paper, we are focusing on a special case that the roofs of prosumers have been rented to PVCO for installing PV panels, and thus the produced PV energy 
belongs to the PVCO. Thus, the prosumers can only act as buyers, and its integral utility function can be formulated as:

$$
U_{n}=k_{n} \ln \left(1+x_{n}\right)-p r \cdot x_{n}
$$

where $U_{n}$ is the integral utility of prosumer $\mathrm{n} ; k_{n}$ is the preference parameter of prosumer $\mathrm{n}$, which changes with its behavioral characteristics; $x_{n}$ is the power consumption of prosumer $\mathrm{n}$ and can be adjusted. We assume that the adjustability of $x_{n}$ results from flexible loads or even battery energy storage systems.

The integral utility of the prosumer increases with the power consumption, whereas the cost of power consumption is also increased. Thus, in order to maximize the integral utility, the prosumer needs to balance between power consumption and cost, and then decides $x_{n}$. It is easy to prove the maximum value of $U_{n}$ is the extreme point of (6). With a given internal price $p r$, the maximal utility of the prosumer can be obtained by:

$$
x_{n}=\frac{k_{n}}{p r}-1
$$

As shown in Figure 4, the integral utility of prosumer is a convex function of power consumption. Moreover, the higher the internal price, the lower the maximal utility of the prosumer with the reduced power consumption.

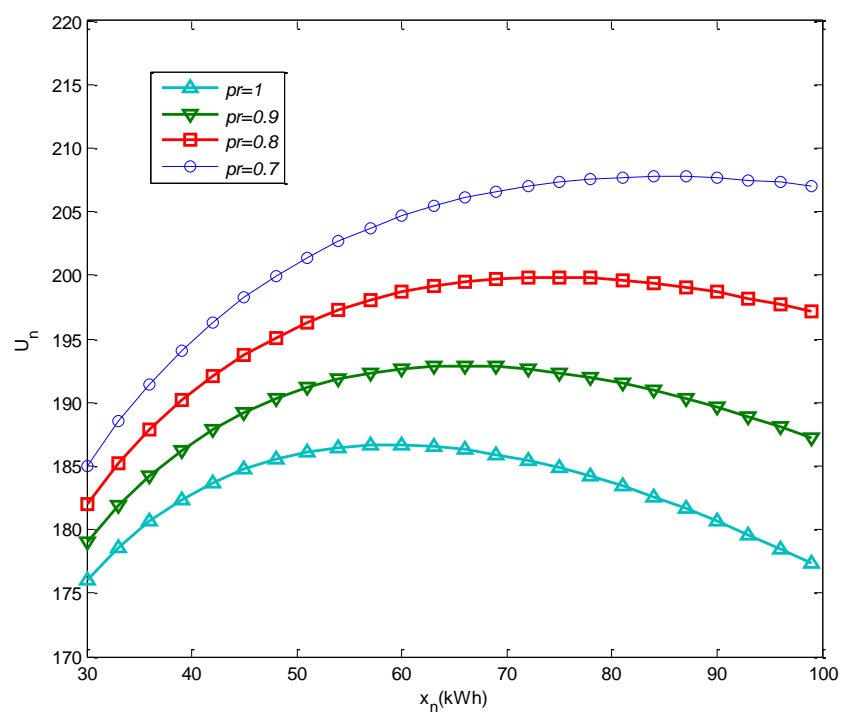

Figure 4. The relationship between prosumer's integral utility and power consumption $\left(k_{\mathrm{n}}=60\right)$.

\section{PVC Demand Response Model}

\subsection{DR Model Based on a Non-Cooperative Game}

The interaction among rational, mutually aware players, where the decisions of some players impact the payoffs of others, is defined as a non-cooperative game. A game, usually denoted by G, is described by its game player, each player's strategies, and the resulting payoffs from each outcome. For example, there are $\mathrm{N}$ game players, the set including all the optional strategies of every game player is called strategy spaces, expressed by $\left\{S_{1}, S_{2} \ldots, S_{N}\right\} . s_{n j} \in S_{n}$ means the j-th strategy of game player $n . u_{n}$ represents the utility of player $n$. Non-cooperative game $G$ of $N$ players is expressed as $\mathrm{G}=\left\{\boldsymbol{S}_{1}, \boldsymbol{S}_{2}, \ldots, s_{N} ; u_{1}, u_{2}, \ldots, u_{N}\right\}$.

The problem of DR in the PVC is essentially a non-cooperative game for prosumers in pursuit of self-utility maximization. Because the internal buying price given by Equation (4) is a function of the total PVC loads, the utility of every prosumer is related with other prosumers' power consumption. For example, when DSR is small at one point, the internal buying price is relatively low. Prosumers 
tend to consume more power in order to promote their utilities. DSR and power cost of this moment both increase because of this power consumption behavior, so every prosumer needs to play the game with others' power consumption arrangement, and searches for the best power consumption. The non-cooperative game of this PVC can be expressed as:

$$
\mathrm{G}_{\mathrm{PVC}}=\left\{\boldsymbol{Y}_{1}, \boldsymbol{Y}_{2}, \ldots, \boldsymbol{Y}_{N} ; U_{1}, U_{2}, \ldots, U_{N}\right\}
$$

where prosumers are the game players, $Y_{n}$ is the available strategy set for prosumer $n, U_{n}$ is the integral utility function of the prosumer $n$.

\subsection{Proof of Nash Equilibrium's Existence and Unique of $G_{P V C}$}

A Nash Equilibrium, named after John Nash, is a set of strategies, one for each player, such that no player has incentive to unilaterally change his/her action [20]. GPVC's Nash Equilibrium is defined as follows:

Definition 1. In the game $G_{P V C}=\left\{Y_{1}, Y_{2}, \ldots, Y_{N} ; U_{1}, U_{2}, \ldots, U_{N}\right\}$, a certain strategy set $\left(x_{1}^{*}, x_{2}^{*}, \ldots, x_{N}^{*}\right)$ composed of one strategy for each game player, and strategy $x_{n}^{*}$ of any game player $n$ is the best strategy of other game players' strategy combination. That is to say, $U_{n}\left(x_{1}^{*}, \ldots, x_{n-1}^{*}, x_{n}^{*}, x_{n+1}^{*}, \ldots, x_{N}^{*}\right) \geqslant$ $U_{n}\left(x_{1}^{*}, \ldots, x_{n-1}^{*}, x_{n}, x_{n+1}^{*}, \ldots, x_{N}^{*}\right)$ is true for any $x_{n},\left(x_{1}^{*}, x_{2}^{*}, \ldots, x_{N}^{*}\right)$ is a Nash equilibrium of $G_{P V C}$.

Theorem 1. When the utility function is strictly quasi-convex function, unique Nash equilibrium exists in the game [21].

For the power consumption $x_{n}$ of any prosumer $n$, there is corresponding power consumption of other prosumers. We use $x_{-n}$ to denote the power consumption of all prosumers except prosumer $n$. Assuming that while prosumer $n$ adjusts its power consumption according to other prosumers' power consumption, the power consumption of other prosumers $x_{-n}$. is fixed. Best response of prosumer $n$ can be obtained by solving:

$$
\max _{n}\left(x_{n}, x_{-n}\right)
$$

As for prosumer $n$ and power consumption of other prosumers, we have:

$$
\begin{gathered}
\operatorname{DSR}\left(x_{n}, x_{-n}\right)=\frac{x_{n}+x_{-n}}{P_{P V}} \\
p r=f(D S R)=\left(\lambda^{b u y}-\lambda^{\text {sell }}\right) e^{\frac{-\alpha P_{P V}}{x_{n}+x_{-n}}}+\lambda^{\text {sell }}
\end{gathered}
$$

Then, the integral utility of prosumer $n$ can be expressed as:

$$
U_{n}=k_{n} \ln \left(1+x_{n}\right)-\left(\lambda^{\text {buy }}-\lambda^{\text {sell }}\right) x_{n} e^{\frac{-\alpha P_{P V}}{x_{n}+x_{-n}}}-\lambda^{\text {sell }} x_{n}
$$

According to (12), under the circumstances that $x_{-n}$ is fixed, the integral utility of prosumer $n$ is the function of $x_{n}$. In order to prove that $U_{n}$ is a strict quasi-convex function of $x_{n}$, the second derivative of $U_{n}$ to $x_{n}$ is needed to be proved less than zero:

$$
\frac{\mathrm{d}^{2} U_{n}}{\mathrm{~d} x_{n}^{2}}=\frac{k_{n}}{\left(1+x_{n}\right)^{2}}-\left(\lambda^{\text {buy }}-\lambda^{\text {sell }}\right)\left(\frac{2 \alpha P_{P V} x_{-n}}{\left(x_{n}+x_{-n}\right)^{3}}+\frac{\alpha^{2} P_{P V}^{2} x_{n}}{\left(x_{n}+x_{-n}\right)^{4}}\right) e^{\frac{-\alpha P_{P V}}{x^{x}+x_{-n}}}
$$

where $k_{n}>0, \lambda^{\text {buy }}>\lambda^{\text {sell }}$ and $\alpha>0$. It can be obtained that the second derivative of $U_{n}$ to $x_{n}$ is less than zero. Then it can be obtained that $U_{n}$ in the GPVC is a strict quasi-convex function. According to Theorem 1, a unique Nash Equilibrium exists in the GPVC. Obviously, in the strategy combination of the Nash Equilibrium, no prosumer is willing to change its power consumption unilaterally. The game 
reaches convergence, and the solution of Nash Equilibrium can be regarded as the optimal power consumption of every prosumer.

\subsection{Nash Equilibrium's Distributed Solution of $G_{P V C}$}

According to Equation (12), under the condition that power consumptions of other prosumers are fixed, the integral utility of prosumer $n$ is the function of its own power consumption. Combined with Definition 1, it can be obtained that optimal strategy of prosumer $n$ is the optimal respond strategy to other prosumers. When every prosumer has reached the optimal power consumption strategy under other prosumers' power consumption strategy, the Nash Equilibrium of game GPVC is reached.

The calculation process is shown in Algorithm 1, where an iterative method was used to solve the Nash Equilibrium of game GPVC. $x_{0}$ is the power consumption corresponding to the utility electricity price $\lambda^{\text {buy }}$ before prosumers participate in the PVC. During the iteration process, PVCO sends the power consumption $x_{-n}$ to prosumer $n$ and receives the optimal power consumption response of prosumer $x_{n}$. All the prosumers are regarded to reach the Nash Equilibrium when the difference of power consumptions between two successive iterations is lower than $\varepsilon$. Finally, the power consumptions of prosumers at this time period have formed the Nash equilibrium strategy of game GPVC.

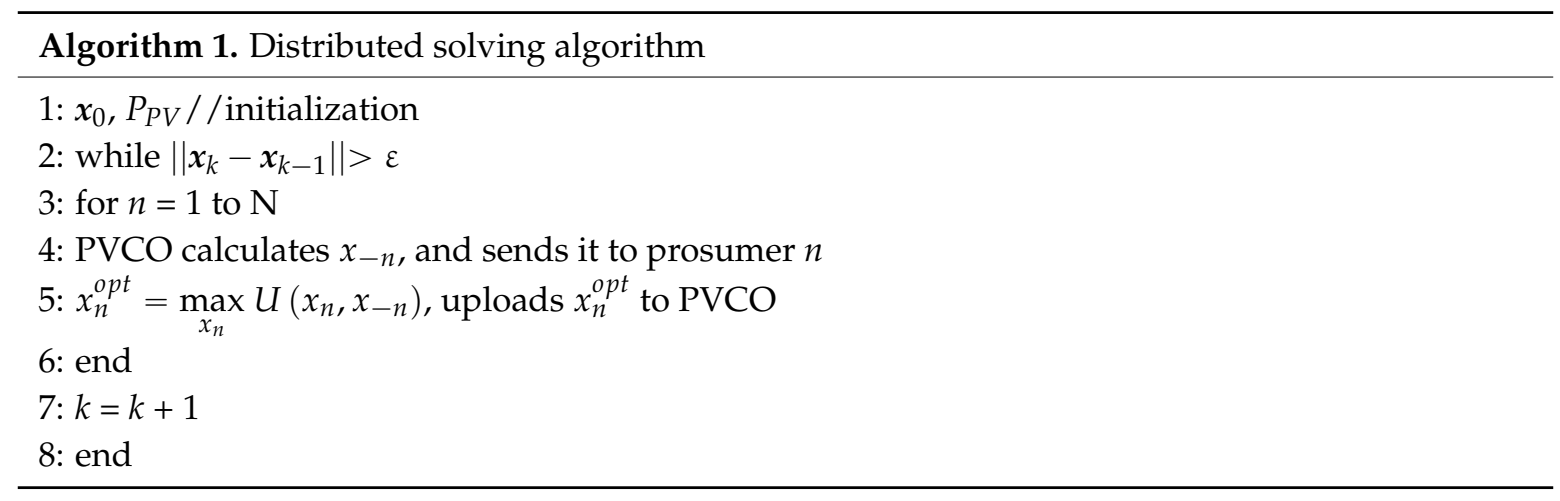

In Algorithm 1, PVCO coordinates the power consumption among prosumers. All prosumers get the $x_{-n}$ instead of the power consumption strategy of a single prosumer, which protects their privacy to some extent. Moreover, by using the distribution algorithm, prosumers can autonomously schedule the optimal power consumption through the acquisition of $x_{-n}$.

\section{Case Study}

\subsection{Basic Data}

A PVC including four prosumers is selected for the case study. Typical power consumptions of these prosumers are shown in Figure 5. Before participating in the PVC, all prosumers consume the power from the utility grid at electricity price $\lambda^{\text {buy }}$. We assume that they achieve their highest utilities at the price $\lambda^{b u y}$, and then the parameter $k_{\mathrm{n}}$ can be obtained by (7). The rated capacity of the PV system in the PVC is $700 \mathrm{kWp}$. The curves of PV energy, total load, and DSR are shown in Figure 6. According to the feed-in tariff of distributed PV sources in China, $\lambda^{\text {sell }}$ is set as the benchmark price of a coal-fired power unit: $0.4 \mathrm{CNY} / \mathrm{kWh}$. $\lambda^{\text {buy }}$ is set as the electricity price of commercial end users, $1.0 \mathrm{CNY} / \mathrm{kWh}$. The parameter of price model $\alpha$ is set as 1 .

As shown in Figures 5 and 6, the load demand of prosumers is relatively high during the time periods 7-18, and PV energy can provide prosumers with cheap electricity. During time periods 7-10 and 15-18, the load of prosumers is greater than the PV energy, whereas in time periods 11-14, the PV energy is at a surplus. 


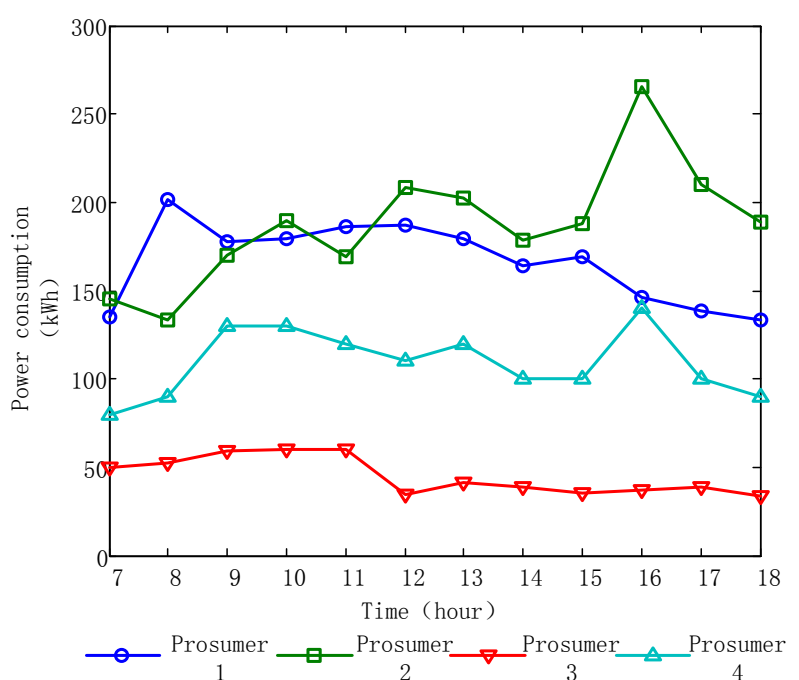

Figure 5. Prosumers' load curves.

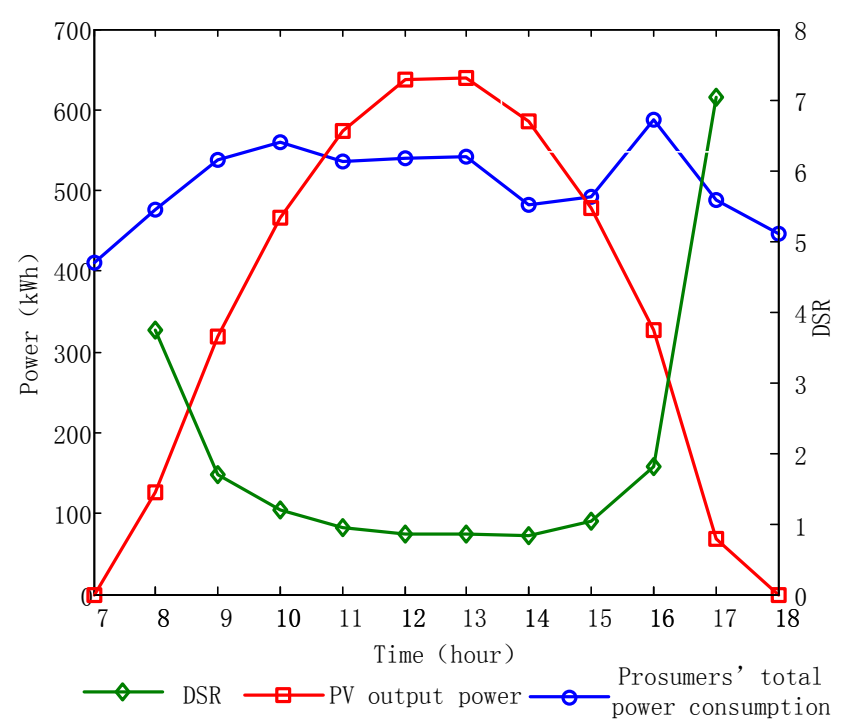

Figure 6. The curves of DSR, PV energy, and total load of prosumers.

\subsection{Local Consumption of PV Energy}

In this section, we assume that the power consumption of prosumers cannot be adjusted. In this regard, we can focus on the reasonability of the internal pricing model. By using models (4) and (5), the internal prices of PVC during time periods 7-18 can be calculated, as shown in Figure 7. According to Figure 6, in time periods $7-10$ and $15-18$, there is DSR $>1$; in time periods $11-14$, there is DSR $<1$. Therefore, in Figure 7, the tendency of internal prices can be divided into three phrases. First, in time periods 7-10, as the DSR (DSR > 1) gradually reduces with the increased PV energy, the internal buying price goes down and the internal selling price rises. Furthermore, in time periods 11-14, as the DSR $(0<$ DSR $<1)$ slowly reduces with the variation of PV energy and load demand, the internal buying price and selling price both go down with the DSR. Finally, in time periods $15-18$, as the DSR (DSR > 1) gradually increases with the decreased PV energy, the internal buying price rises, but the internal selling price goes down. For all the time periods, the internal prices have always been conformed to the constraint $\lambda^{\text {sell }} \leqslant p r_{P V} \leqslant p r \leqslant \lambda^{\text {buy }}$. 


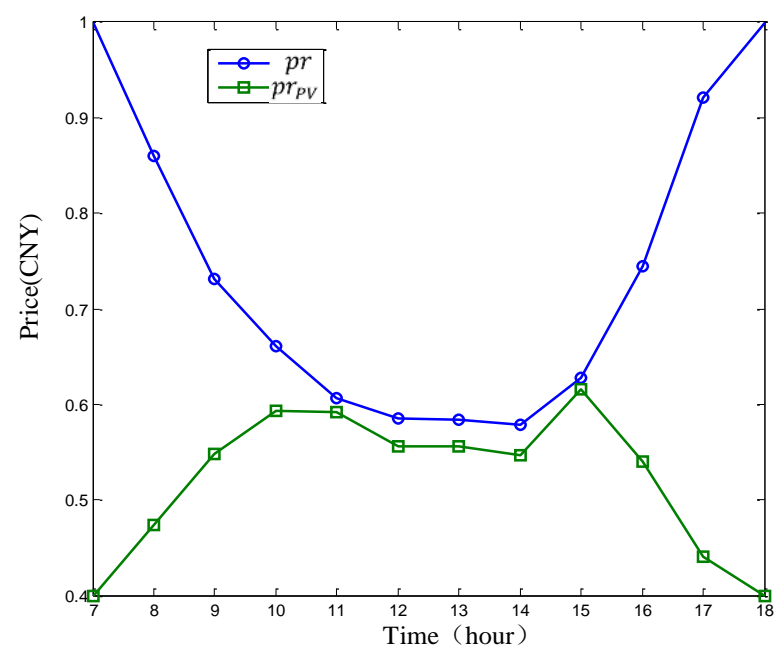

Figure 7. Internal prices of the PVC.

Moreover, the integral utilities of prosumers and the benefit of PVCO can be obtained based on the internal prices and original power consumptions, as shown in Figures 8 and 9. The results show that the internal prices of PVC are able to increase the integral utilities of all prosumers and the benefit of the PVCO, even if the prosumers have no flexible loads or battery storage systems.

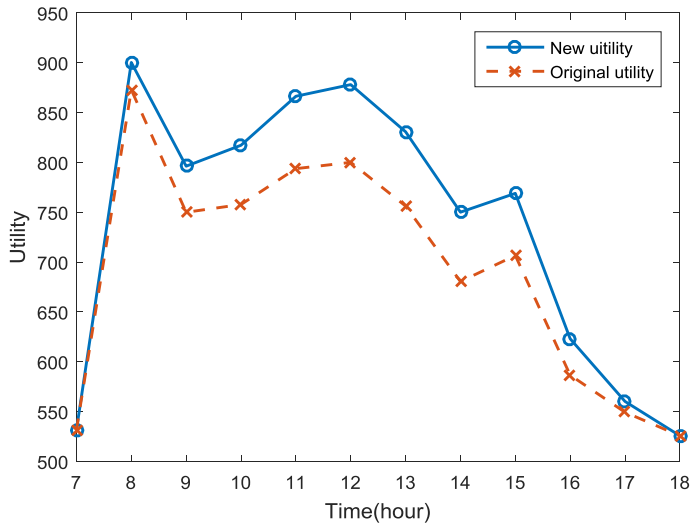

(a)

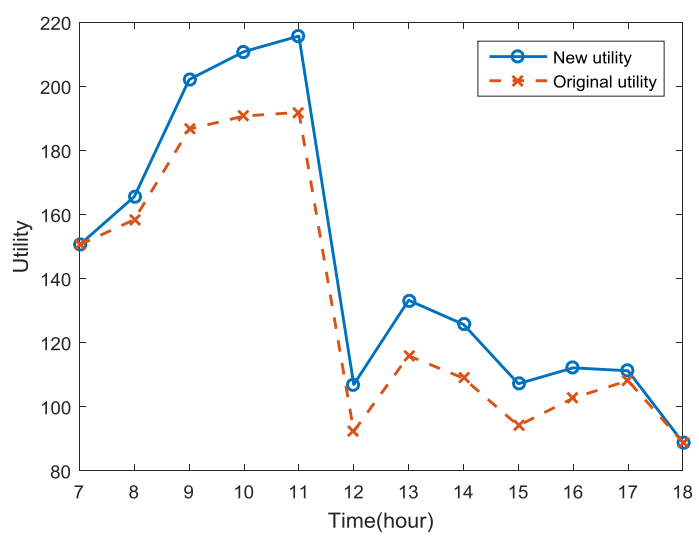

(c)

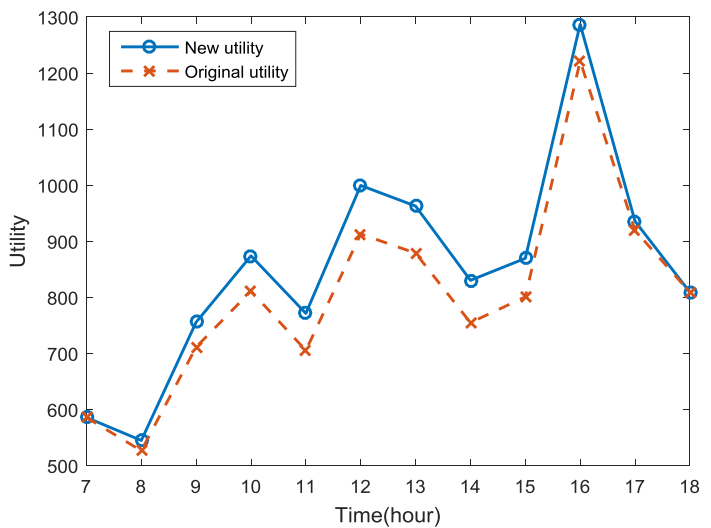

(b)

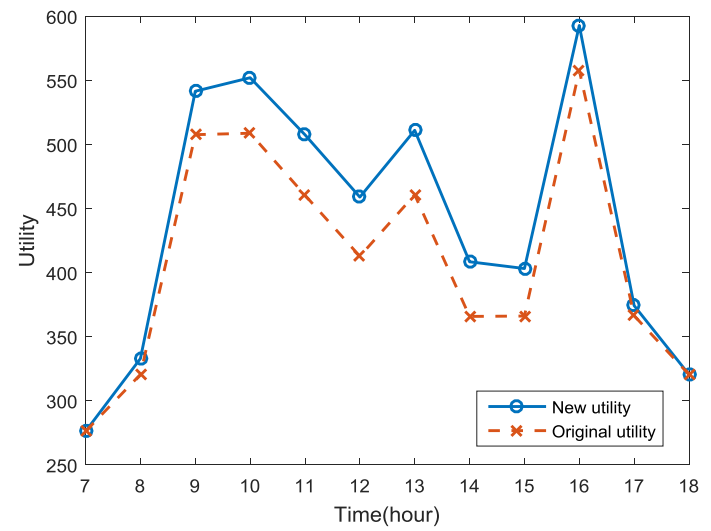

(d)

Figure 8. The comparison of the integral utilities of prosumer 1 (a); prosumer 2 (b); prosumer 3 (c) and prosumer 4 (d) before and after participating the PVC. 


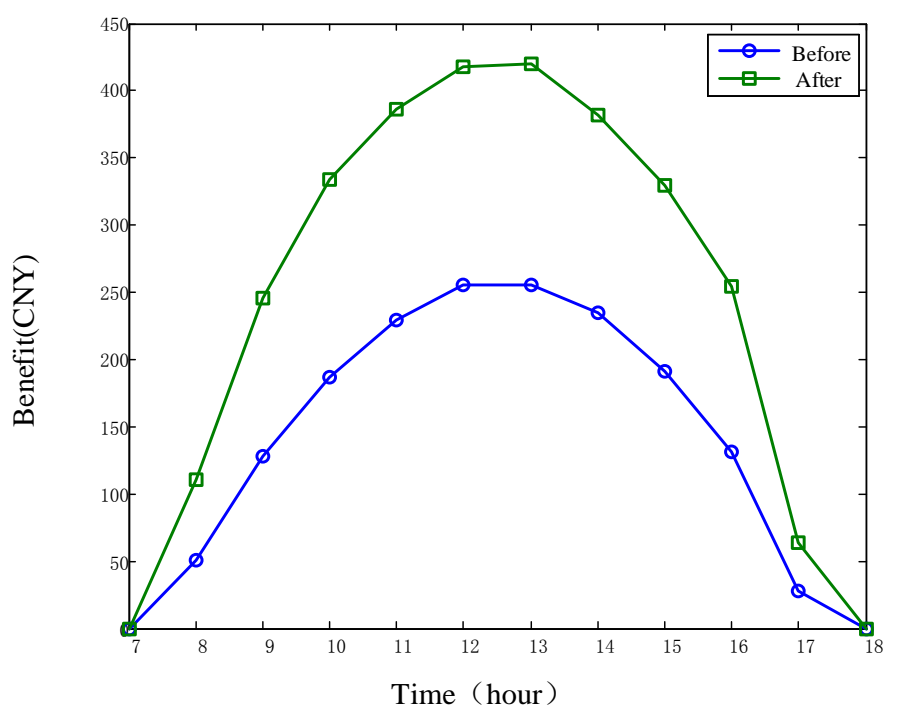

Figure 9. Comparison of the PVCO's benefit of selling PV electricity before and after participating in the PVC.

In order to verify that the internal pricing model can guarantee the economic balance of the PVC, the total power consumption of prosumers, PV energy, total power purchase fee, the benefit of PVCO, and NEC are shown in Table 1. The results show that the total power purchase fees of prosumers always equals the sum of the PVCO's benefit and NEC.

Table 1. Incomes and expenditures of participant entities in the PVC.

\begin{tabular}{cccccc}
\hline Time (h) & $\begin{array}{c}\text { PV Output } \\
\text { Power (kWh) }\end{array}$ & $\begin{array}{c}\text { Prosumers' Total } \\
\text { Consumption (kWh) }\end{array}$ & $\begin{array}{c}\text { Prosumers' Total } \\
\text { Fee (CNY) }\end{array}$ & $\begin{array}{c}\text { PVCO's } \\
\text { Benefit (CNY) }\end{array}$ & NEC (CNY) \\
\hline 7 & 0 & 410.35 & 410.35 & 0 & 410.35 \\
8 & 127.26 & 467.91 & 409.90 & 160.24 & 249.66 \\
9 & 318.78 & 537.10 & 392.85 & 174.53 & 218.32 \\
10 & 466.20 & 559.03 & 369.30 & 276.46 & 92.83 \\
11 & 573.30 & 535.81 & 324.60 & 339.60 & -15.00 \\
12 & 637.56 & 540.83 & 316.16 & 354.85 & -38.69 \\
13 & 640.08 & 542.60 & 317.11 & 356.10 & -39.00 \\
14 & 585.90 & 482.24 & 278.75 & 320.22 & -41.46 \\
15 & 477.54 & 492.13 & 308.75 & 294.16 & 14.59 \\
16 & 327.60 & 588.42 & 437.69 & 176.87 & 260.82 \\
17 & 69.30 & 478.26 & 448.50 & 30.54 & 417.96 \\
18 & 0 & 446.26 & 446.26 & 0 & 446.26 \\
\hline
\end{tabular}

\subsection{Analysis of $D R$}

In this section, we assume that every prosumer has the ability to adjust its power consumption according to the internal prices. By using Algorithm 1, iterative processes of the prosumers are shown in Figure 10.

The results show that the iterative process of each prosumer can be completed within 5 generations with high efficiency. Before and after the DR, the comparisons of the internal prices and prosumers' utilities in time periods 7-18 are shown in Figures 11 and 12, respectively. 


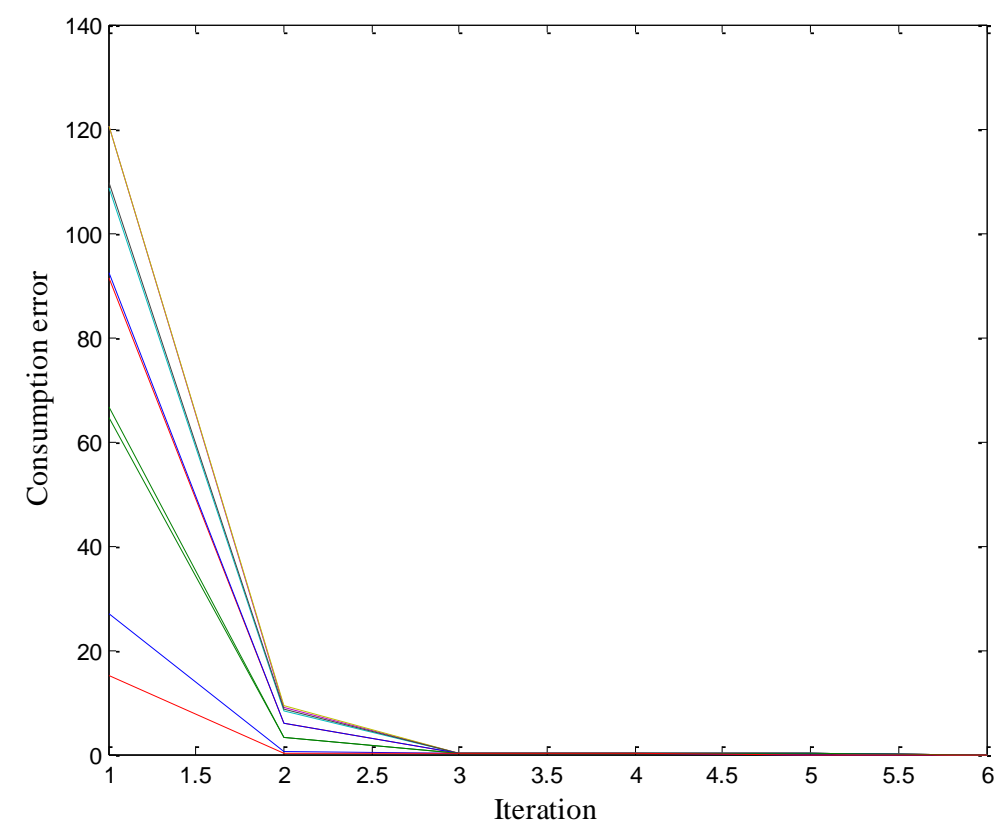

Figure 10. The convergence of the prosumers' electricity consumption based on a non-cooperative game.

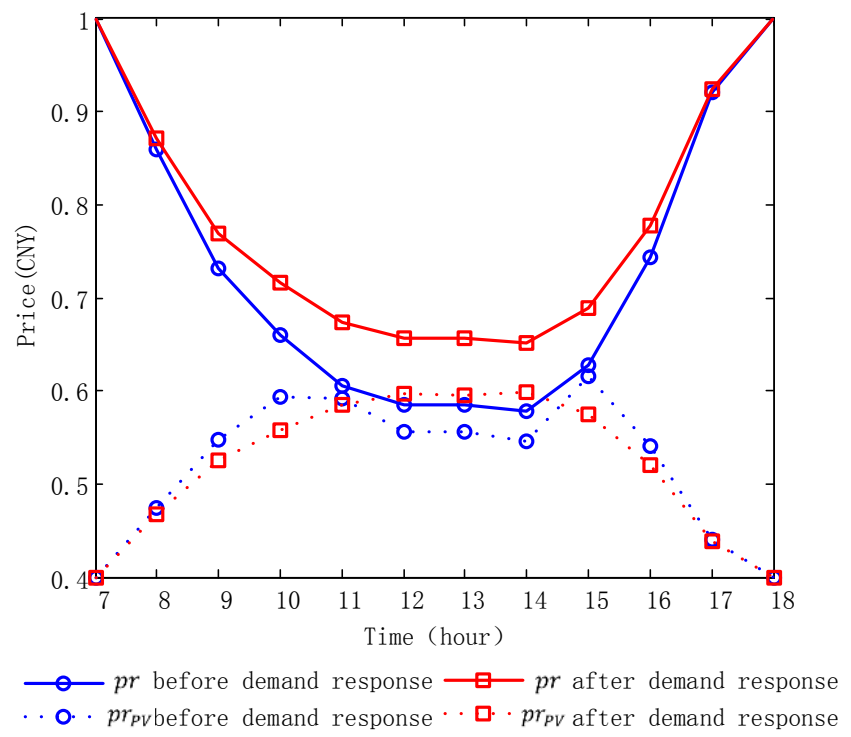

Figure 11. The comparison of internal prices before and after DR.

In Figure 11, the results show that the internal prices are increased with the DR of prosumers, due to the incentive of cheap PV energy supply. As the PV energy is constant, the DSR is increased with the total power consumption of prosumers. Thus, the internal buying price will be increased accordingly. Moreover, for the PVCO, the internal selling prices would be maximized when the DSR is getting close to 1 . Thus, when in time periods 7-10 and 15-18 (DSR > 1), the internal selling price goes down with the increased DSR. In contrast, in time periods 12-14 (DSR $<1)$, the internal selling price rises with the increased DSR. 


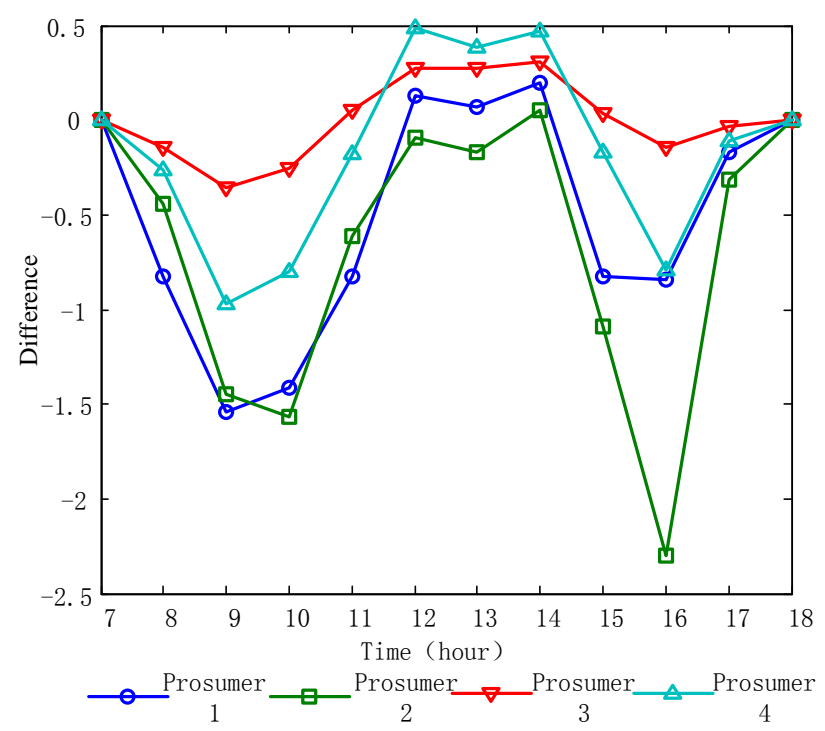

Figure 12. The comparison of prosumers' utilities before and after DR.

In order to analyze the change of utilities after the prosumers participate in DR, difference values of prosumers' utilities in each time period are shown in Figure 12. The results show that the utilities of prosumers have been reduced in time periods 7-10 and 15-18 (DSR > 1), whereas they increased in time periods $12-14$ (DSR $<1$ ). This is an interesting result, and the reason is that when DSR $>1$, the non-cooperative game will push prosumers to consume more energy, and thus increase the internal buying price. Although more power consumption can accordingly increase the utilities of prosumers, the increment of internal buying price has a greater negative influence on their integral utilities. Moreover, in time periods 12-14 (DSR < 1), even though the internal buying price has been increased with the power consumptions of prosumers, their utilities still can be increased with more consumptions in general.

Having insight into this result, when the PV energy is at a surplus, it seems more appropriate to use DR, and thus the integral utilities of prosumers and the benefit of PVCO can be improved simultaneously. Whereas the PV energy is insufficient, a vicious competition among prosumers will eventually decrease the prosumers' integral utilities and the benefit of PVCO. In order to avoid the vicious competition, the DR can be conditionally used during the time periods of DSR $<1$, only if the internal prices are lower than a certain value.

\subsection{Sensitivity Analysis of $\alpha$}

According to the analysis of Section 3.2, $\alpha$ is defined as the adjustment parameter for deciding the price level. That is, the PVCO can use $\alpha$ to adjust the internal prices according to different circumstances. Generally, when DSR is constant, the greater $\alpha$, the lower the internal prices are. For both benefits of prosumers and PVCO, they need to negotiate for the setting. Thus, we assume that $\alpha$ is 0.5 and 1 , the internal prices and the integral utilities of prosumers are shown in Figures 13 and 14.

In Figures 13 and 14, the results of internal prices are consistent with the analysis of $\alpha$ shown in Figure 3. If $\alpha=0.5$, the internal prices are more beneficial to the PVCO. Otherwise, if $\alpha=1$, the internal prices are more beneficial to the prosumers. From the basic design principles of the internal pricing model, any value of $\alpha$ in the internal of $(0,1]$ is reasonable, which can be beneficial to both prosumers and PVCO, as compared to trading with the utility grid. However, the different value of $\alpha$ can lead to a different benefit distribution of the produced PV energy. In practical applications, we think it is important to consider the contributions to investment in PV systems. If the PVCO invests in all the PV systems, it is apparent that the $\alpha$ value should be set to a smaller value to increase the Return of Investment (ROI). Considering the feed-in-tariff, utility electricity price, and the investment cost in 
China [22], we think it is reasonable to set the $\alpha$ between 0.5 and 0.3 , and thus the ROI period would be $6-8$ years. This is a decent result for investors in renewable energy.

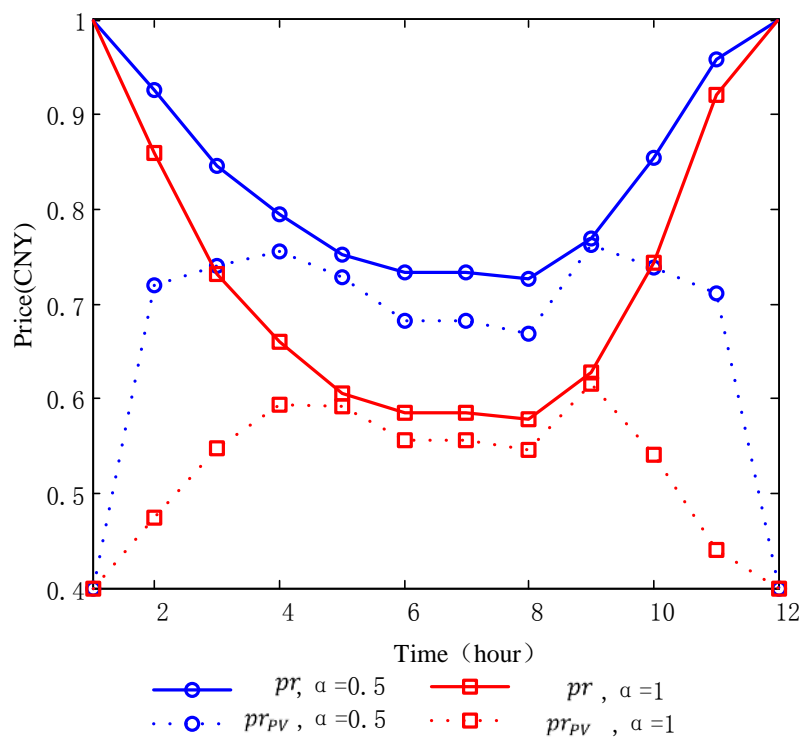

Figure 13. Impact of $\alpha$ on internal price in PVC.

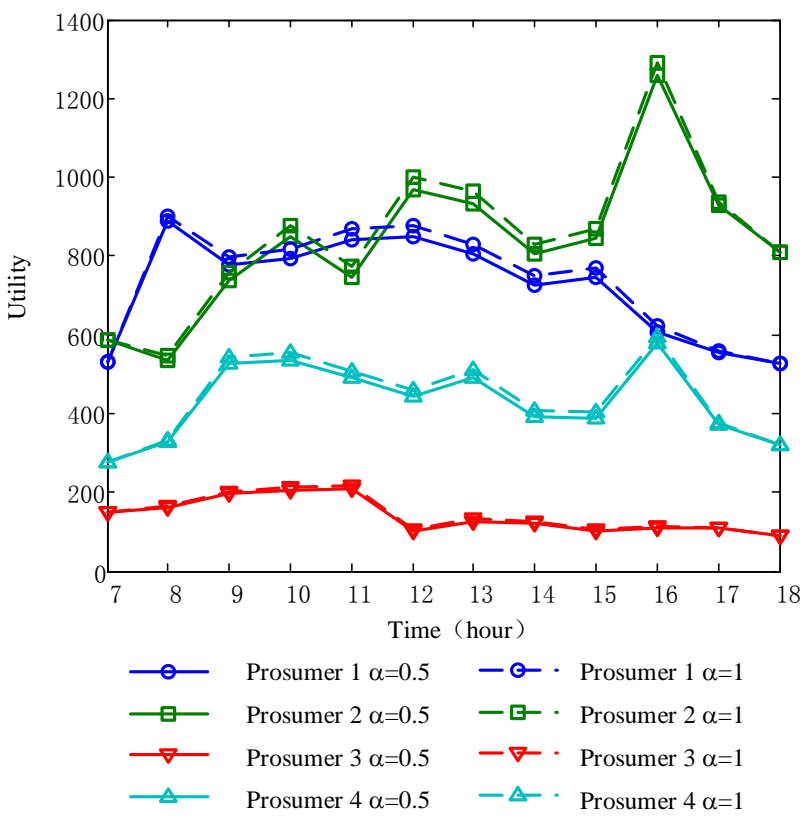

Figure 14. Impact of $\alpha$ on prosumers' utility.

\section{Conclusions}

In this paper, we have proposed a multi-party energy management for PV clusters of leased-roof prosumers. According to the economic principle of demand and supply, we have formulated a dynamic internal pricing model for the PVCO and prosumers. We have shown that the internal pricing model can simultaneously promote the integral utilities of prosumers and the benefit of PVCO. Moreover, if the prosumers have flexible load or battery energy storage system resources, the DR can be used in the PVCO to improve the local consumption of PV energy. We have formulated the DR problem in the PVC as a non-cooperative game among prosumers, and proved the existence and uniqueness of the Nash Equilibrium. We have also shown that the DR is effective when the PV energy is at a surplus. 
Finally, we have investigated the sensitivity of the adjustment parameter of the price level. According to China's energy policy and the cost of investment, a reasonable value for $\alpha$ to be set is between 0.5 and 0.3 .

The proposed work can be extended in various ways. For instance, if pure consumers, which have no roofs for leasing, would like to participate in the PVC, the pricing model should be discriminated [23], since their contributions to the PVC are not as obvious as those of the roof-leasing prosumers. Furthermore, we can also discuss the crowdfunding model for PV systems. Although the consumers cannot offer their roofs for leasing, they can still participate in the PVC by a crowdfunding mechanism of investment. Another interesting extension is the sizing and energy management of battery storage systems. For the PVC, the battery storage can be configured centralized with the PVCO or distributed with each prosumer. The effectiveness and the related energy management strategies need to be investigated in the future.

Acknowledgments: This work was supported by the National High Technology Research and Development Program of China (863 Program) (2014AA052001).

Author Contributions: The paper was a collaborative effort between the authors. Nian Liu, Cheng Wang and Xinhao Lin contributed collectively to the theoretical analysis, modeling, simulation, and manuscript preparation. Jinyong Lei prepared the manuscript and improved this manuscript's English language and style.

Conflicts of Interest: The authors declare no conflict of interest.

\section{Appendix}

According to three basic principles: (i) the internal prices should be bounded between the feed-in tariff and electricity prices of the utility grid; (ii) PV power should be utilized in the PVC as much as possible, the dynamic pricing model can be used to encourage the consumption when the PV power is at a surplus; (iii) the economic balance should always be guaranteed by PVCO.

Thus, for prosumers, the internal buying price would be raised with the increased DSR, and should not be higher than $\lambda^{\text {buy }}$, even with the condition DSR $\rightarrow \infty$. To meet these principles, the internal price can be set in accordance with the function of $a e^{\frac{-\alpha}{x}}+b$ (in which $x$ is an independent variable). Thus, we can use $a e^{\frac{-\alpha}{x}}+b$ as the fitting function to obtain the relationship between internal price and DSR:

$$
p r=f(D S R)=a e^{\frac{-\alpha}{D S R}}+b
$$

According to the definition of DSR, when the total power consumption is much lower than the PV energy (DSR $\rightarrow 0$ ), the surplus PV energy has to be fed to the utility grid. To encourage prosumers to consume more power, the internal price should be set at a lower bound $\lambda^{\text {sell }}$. Thus, the function $f(D S R)$ should include the point $\left(0, \lambda^{\text {sell }}\right)$. Furthermore, when the total power consumption is much higher than the PV energy (DSR $\rightarrow+\infty$ ), the energy needed by prosumers is bought from the utility grid at the price of $\lambda^{\text {buy }}$. To regulate prosumers' consumption, the internal price should be set as the higher bound $\lambda^{b u y}$, so the point $\left(+\infty, \lambda^{b u y}\right)$ is also included in the function $f(D S R)$. By substituting these two points into the function $f(D S R)$, we can further obtain the following equation set:

$$
\left\{\begin{array}{c}
b=\lambda^{\text {sell }} \\
a+b=\lambda^{\text {buy }}
\end{array}\right.
$$

Solving the equation set, we can obtain:

$$
\left\{\begin{array}{c}
a=\lambda^{\text {buy }}-\lambda^{\text {sell }} \\
b=\lambda^{\text {sell }}
\end{array}\right.
$$


Finally, by substituting (16) into (14), the function of internal selling price can be formulated as:

$$
p r=f(D S R)=\left(\lambda^{\text {buy }}-\lambda^{\text {sell }}\right) e^{\frac{-\alpha}{D S R}}+\lambda^{\text {sell }}
$$

Considering the economical balance requirement of the PVC, we can deduce the PV power selling price $p r_{P V}$ based on internal price $p r$.

If $0 \leqslant D S R \leqslant 1$, the PVCO needs to sell the surplus PV energy to the utility grid at the price of $\lambda^{\text {sell }}$, and the economic balance can be described as:

$$
\sum_{n=1}^{N} x_{n} \cdot p r+\left(P_{P V}-\sum_{n=1}^{N} x_{n}\right) \lambda^{\text {sell }}=P_{P V} \cdot p r_{P V}
$$

By substituting (3) into (A5), we can deduce the $p r_{P V}$ as:

$$
\operatorname{pr}_{P V}=D S R \cdot p r+(1-D S R) \lambda^{\text {sell }}
$$

Otherwise, if DSR $>1$, the PVCO needs to buy the energy from the utility grid at the price of $\lambda^{\text {buy }}$, and the economic balance can be described as:

$$
\sum_{n=1}^{N} x_{n} \cdot p r=P_{P V} \cdot p r_{P V}+\left(\sum_{n=1}^{N} x_{n}-P_{P V}\right) \lambda^{b u y}
$$

By substituting (3) into (A7), we can deduce the $\operatorname{pr}_{P V}$ as:

$$
\operatorname{pr}_{P V}=D S R \cdot p r-(D S R-1) \lambda^{b u y}
$$

Finally, the function of PV power selling price can be formulated as:

$$
\operatorname{pr}_{P V}=g(D S R)=\left\{\begin{array}{l}
p r \cdot D S R+\lambda^{\text {sell }}(1-D S R), 0 \leqslant D S R \leqslant 1 \\
p r \cdot D S R-\lambda^{\text {buy }}(D S R-1), D S R>1
\end{array}\right.
$$

\section{References}

1. Canova, A.; Giaccone, L.; Spertino, F.; Tartaglia, M. Electrical impact of photovoltaic plant in distributed network. IEEE Trans. Ind. Appl. 2009, 45, 341-347. [CrossRef]

2. Alam, M.J.E.; Muttaqi, K.M.; Sutanto, D. A SAX-based advanced computational tool for assessment of clustered rooftop solar PV impact on LV and MV networks in smart grid. IEEE Trans. Smart Grid 2013, 4, 577-585. [CrossRef]

3. Kanchev, H.; Colas, F.; Lazarov, V.; Francois, B. Emission reduction and economical optimization of an urban microgrid operation including dispatched PV-based active generators. IEEE Trans. Sustain. Energy 2014, 5, 1397-1405. [CrossRef]

4. Meng, F.; Zeng, X. A Stackelberg game-theoretic approach to optimal real-time pricing for the smart grid. Soft Comput. 2013, 17, 2365-2380. [CrossRef]

5. Maharjan, S.; Zhu, Q.; Zhang, Y.; Gjessing, S.; Basar, T. Dependable demand response management in the smart grid: A Stackelberg game approach. IEEE Trans. Smart Grid 2013, 4, 120-132. [CrossRef]

6. McKenna, E.; Thomson, M. Photovoltaic metering configurations, feed-in tariffs and the variable effective electricity prices that result. IET Renew. Power Gen. 2013, 7, 235-245. [CrossRef]

7. Ding, Z.; Lee, W.; Wang, J. Stochastic resource planning strategy to improve the efficiency of microgrid operation. IEEE Trans. Ind. Appl. 2015, 51, 1978-1986. [CrossRef]

8. Chiu, W.; Sun, H.; Poor, H.V. Energy imbalance management using a robust pricing scheme. IEEE Trans. Smart Grid 2013, 4, 896-904. [CrossRef]

9. Fathi, M.; Bevrani, H. Statistical cooperative power dispatching in interconnected microgrids. IEEE Trans. Sustain. Energy 2013, 4, 586-593. [CrossRef] 
10. Liu, X.; Wang, Z.; Gao, F.; Guan, X.; Zhai, Q.; Wu, J. Internal pricing mechanism for hierarchical power consumption/generation scheduling in industrial enterprise microgrid. In Proceedings of the IEEE PES Asia-Pacific Power and Energy Engineering Conference (APPEEC), Kowloon, Hong Kong, 8-11 December 2013.

11. Lin, X.; Wang, Y.; Pedram, M. Designing the optimal pricing for aggregators in the smart grid. In Proceedings of the Sixth Annual IEEE Green Technologies Conference (GreenTech), Corpus Christi, TX, USA, 3-4 April 2014.

12. Maity, I.; Rao, S. Simulation and pricing mechanism analysis of a solar-powered electrical microgrid. IEEE Syst. J. 2010, 4, 275-284. [CrossRef]

13. Tushar, W.; Chai, B.; Yuen, C.; Smith, D.B.; Wood, K.L.; Yang, Z.; Poor, H.V. Three-party energy management with distributed energy resources in smart grid. IEEE Trans. Ind. Electron. 2015, 62, 2487-2498. [CrossRef]

14. Tushar, W.; Chai, B.; Yuen, C.; Huang, S.; Smith, D.; Poor, H.V.; Yang, Z. Energy Storage Sharing in Smart Grid: A Modified Auction Based Approach. IEEE Trans. Smart Grid 2016, 7, 1462-1475. [CrossRef]

15. Ma, L.; Liu, N.; Wang, L.; Zhang, J.; Lei, J.; Zeng, Z.; Wang, C.; Cheng, M. Multi-Party Energy Management for Smart Building Cluster with PV Systems Using Automatic Demand Response. Energy Build. 2016, 121, 11-21. [CrossRef]

16. Bie, Z.; Xie, H.; Hu, G.; Li, G. Optimal scheduling of power systems considering demand response. J. Mod. Power Syst. Clean Energy 2016, 4, 180-187. [CrossRef]

17. Liu, N.; Chen, Q.; Liu, J.; Lu, X.; Li, P.; Lei, J.; Zhang, J. A heuristic operation strategy for commercial building microgrids containing EVs and PV system. IEEE Trans. Ind. Electron. 2015, 62, 2560-2570. [CrossRef]

18. Liu, N.; Chen, Q.; Lu, X.; Liu, J.; Zhang, J. A Charging Strategy for PV-Based Battery Switch Stations Considering Service Availability and Self-Consumption of PV Energy. IEEE Trans. Ind. Electron. 2015, 62, 4878-4889. [CrossRef]

19. Gans, J.; King, S.; Stonecash, R.; Mankiw, N.G. Principles of Economics; Cengage Learning: Boston, MA, USA, 2011.

20. Nash, J.F. Equilibrium points in a-person games. Proc. Natl. Acad. Sci. USA 1959, 27, 54-71. [CrossRef]

21. Resen, J.B. Existence and uniqueness of equilibrium points for concave n person games. Econometrica 1965, 33, 520-534. [CrossRef]

22. Tan, Z.; Chen, K.; Ju, L.; Liu, P.; Zhang, C. Issues and solutions of China's generation resource utilization based on sustainable development. J. Mod. Power Syst. Clean Energy 2016, 4, 147-160. [CrossRef]

23. Tushar, W.; Yuen, C.; Smith, D.B.; Poor, H.V. Price discrimination for energy trading in smart grid: A game theoretic approach. IEEE Trans. Smart Grid 2016. [CrossRef] 